\title{
Éditorial
}

\section{Pratique innovante et théorie déficiente : formalisation vs. privatisation dans la question de la résolution de conflits}

Que de conflits dans cette deuxième édition de la nouvelle RCrit! En fin de compte, notre monde actuel qui, selon les points de vue, s'étend ou s'effrite, mais qui de toute façon se mondialise, couve lui aussi des conflits à outrance. Rien que les instruments juridiques qui permettraient de les résoudre sont fréquemment jugés insuffisants. Ainsi, par exemple, les procédures des autorités et tribunaux administratifs ne semblent pas (ou plus) être un moyen efficace d'apaiser, de manière durable et satisfaisante pour chacun, les litiges relatifs à la réalisation de projets d'envergure - tels l'aménagement de gares, la mise en service d'aéroports ou l'extension de réseaux électriques -, dont les répercussions seraient ressenties de manière égale aux niveaux local et national. Dans ce cas, il est possible que les procédures de conciliation et les éléments plébiscitaires de la formation démocratique de la volonté engendrent des résultats plus rapidement et de façon plus efficace. Même la justice pénale et la justice civile se retrouvent confrontées à des démarches alternatives vers un règlement des litiges. Celles-ci sont issues d'une pratique du droit innovante et élargissent voire abandonnent le cadre des procédures traditionnelles et normalisées, qu'il s'agisse d'une transaction, d'une adjudication ou d'une médiation. Mais ces alternatives ne sont pas non plus exemptes de problèmes, en particulier lorsqu'on touche aux questions de l'État de droit ou de la faisabilité du droit. Les réactions du monde politique (juridique), notamment du pouvoir législatif, telles qu'elles se manifestent par exemple dans le cadre de la directive relative à la médiation dans les États membres de l'UE et de son application par ces mêmes États membres, semblent, à ce stade, plutôt logiques. Les tendances à la privatisation et à la formalisation se font face dans une structure dynamique et sont fréquemment reliées l'une à l'autre. La manière dont les modifications s'opèrent au sein de cette structure est, par contre, fréquemment jugée déficiente, et a fortiori lorsqu'il s'agit de la fondation théorique.

Depuis le début de l'année 2012, ce déficit est au cœur de l'axe de recherche "Résolution extrajudiciaire et judiciaire de conflits » à Francfort-sur-le-Main (www.konfliktloesung.eu). Ce dernier a été initié conjointement par l'Université Johann Wolfgang Goethe, l'Institut Max Planck d'Histoire juridique européenne (MPIeR), l'école supérieure spécialisée de Francfort-sur-le-Main - University of Applied Sciences et, en sa qualité de partenaire associé, la Gesellschaft für Reichskammergerichtsforschung e.V. ${ }^{1}$ (Wetzlar). Il est financé dans le cadre du programme LOEWE (offensive du Land de Hesse pour le développement d'une excellence scientifico-économique), pour trois ans, à hauteur de 3,4 millions d'euros en tout. Grâce à une approche polychrone et comparative et à la coordination de l'histoire juridique, la science juridique, la science historico et la sinologie, diverses formes du règlement et de la résolution de conflits sont analysées sous un angle historique-empirique, ce qui engendre une con-

1 Société pour la recherche sur la cour de la chambre impériale. 
naissance structurelle. Afin de mettre cette connaissance directement à la disposition du discours social, un conseil d'experts formé de praticiens du règlement extrajudiciaire et judiciaire de litiges travaille de façon innovante au transfert de la connaissance du monde académique à la société.

Un motif suffisant, aux yeux de la RCrit, d'accorder ne fût-ce qu'un peu d'attention aux activistes LOEWE de Francfort : un atelier de l'axe de recherche, organisé par Peter Collin au MPIeR au mois de février dernier, intitulé « Concilier et résoudre. Différenciation et hybridation », a prouvé jusqu'à quel point la communication entre la science et la pratique peut aboutir, dans un cadre théorique de référence. Quatre contributions du présent numéro sont justement issues de cette manifestation : Martin Engel, assistant scientifique à l'Université Ludwig Maximilian de Munich, décrit les critères qualitatifs et les propositions de modèle d'une résolution de conflits consensuelle - c'est-à-dire basée sur une réflexion commune des parties en litige et de leurs avocats - en regard de la nécessité et de l'ampleur de leur règlement au niveau étatique. Sa réponse émet une certaine réserve à l'égard du modèle souhaité proche d'un multi-door-courthouse, au profit d'une garantie de la qualité des conseils juridiques, basée sur des stimulants économiques. Pour Karl Härter (MPIeR), professeur à l'Université technique de Darmstadt, l'infrajustice, en tant que concept de la recherche historique de la criminalité, ne représente certes pas encore un modèle théorique parfait, mais pourrait bien contribuer à modifier le terme résolution de conflits (orienté vers les résultats) et à lui préférer les termes arrangement ou règlement de conflits, ainsi qu'à vaincre la réduction de la dichotomie alternative « judiciaire / extrajudiciaire ». Patrick Schroeder, avocat et médiateur économique à Francfort-sur-le-Main, se consacre à la juridiction de conciliation, comme pendant à la juridiction d'État. L'attestation d'une alternative efficace et parfois privilégiée au règlement de litiges, qu'il délivre à la juridiction de conciliation, ne diminue pas la nécessité de s'adapter aux besoins variables des personnes ayant recours aux services de règlement des litiges.

Trois autres contributions de cette édition, si elles ne proviennent pas du contexte de LOEWE, contiennent toutefois autant de matière sur la question des conflits : Sarah Henneberger-Sudjana et Fred Henneberger, de l'Institut de recherche pour le travail et le droit du travail de l'Université de St. Gallen, comparent l'évolution actuelle du droit de grève du service public en Suisse et en Allemagne. Leur comparaison avec la situation juridique des deux pays aboutit à la même conclusion que l'analyse d'Anja Schiemann, professeure privée et avocate à Francfort-sur-le-Main, portant sur le droit des prévenus dans le droit de procédure pénale actuel, comparé à la position juridique de la victime au cours de la procédure : le législateur doit agir. Halina Wazyniak, députée du Bundestag, soulève la même revendication à propos de la requête d'un accès à Internet dans le système carcéral. Jusqu'à présent, cette requête n'a été enregistrée et appliquée que de façon incomplète et insuffisante, en vertu de la loi de chaque Land, à la suite de la réforme du fédéralisme. Un recueil historico-scientifique réalisé à l'occasion du $200^{\mathrm{e}}$ anniversaire de la fondation de l'Université Humboldt de Berlin offre un aperçu de l'histoire de la faculté de droit de l'établissement, riche en conflits. Yorick Wirth, collaborateur scientifique dans l'axe de recherche LOEWE « Résolution extrajudiciaire et judiciaire de conflits », en fait la critique pour RCrit.

À l'instar d'un éditorial, la recherche historique et contemporaine reste incomplète si elle ne tourne pas un regard vers l'avenir : la Journée de la politique juridique 2012, la 
$21^{\text {e }}(!)$, organisée par le département spécialisé de sciences juridiques de l'Université Goethe, en coopération avec le ministère de la Justice, de l'intégration et de l'Europe de Hesse, est également consacrée à des formes alternatives de règlement de litiges et de résolution de conflits. C'est pourquoi, chère lectrice, cher lecteur, nous vous recommandons, à vous, Européens convaincus et dotés d'un esprit critique, de célébrer ce 27 novembre 2012!

Francfort-sur-le-Main, juin 2012

Guido Pfeifer 